\title{
Analysis and Experiment of Eddy Current Loss in Radial Magnetic Bearings with Solid Rotor
}

\author{
Zengyuan Yin ${ }^{1}$, Yuanwen Cai ${ }^{1}$, Weijie Wang ${ }^{1}$ and Yuan Ren ${ }^{1}$ \\ ${ }^{1}$ Space Engineering University, Beijing 101416, China
}

\begin{abstract}
In order to reduce power losses for the aerospace applications, this paper analyzes the eddy current losses produced by high-speed rotating solid rotor core magnetic bearings of magnetically suspended control \& sensitive gyroscope (MSCSG). An analytical model of the eddy current loss of a solid rotor radial magnetic bearing (RMB) is presented. Considering the difference between NNNN and NSNS magnetic circuits of RMB, the magnetic field expressions of stator magnetic poles are listed. The magnetic field of stator poles is replaced by Fourier series expansion. According to the magnetic field distribution around the magnetic pole surface and the boundary conditions of the rotor surface, the mathematical expression of eddy current loss is obtained. The measurement method of rotational power loss in radial magnetic bearing is proposed, and the results of the theoretical analysis are verified by experiments in the prototype MSCSG. The experimental results show the correctness of calculation results. Eddy current loss models and test methods provide theoretical support for analyzing eddy current losses in solid rotors and reducing power consumption.
\end{abstract}

\section{Introduction}

Active magnetic bearings can eliminate the friction of traditional mechanical bearings, no lubrication, high torque accuracy, micro-vibration, active vibration suppression and other advantages [1-2]. Not only the output torque accuracy improve by 1 2 orders of magnitude compared to the mechanical bearing flywheel, but also has a long life. Therefore, the inertial actuator supported by the magnetic bearing is an ideal inertial actuator for the high-precision earth observation satellite. When the rotor used for the magnetic bearing support rotates at a high speed, in addition to the friction caused by the air friction, the rotor will also generate eddy current loss and hysteresis loss. Generally, the eddy current loss is far greater than the hysteresis loss, which seriously affects the inertial actuator performance. In order to reduce the eddy current loss, the laminated structure is usually adopted, but in some cases, a solid rotor is still needed. The focus of the research in this paper is to analyze and experimentally verify the eddy current loss of the magnetic bearing with the solid rotor.

Eddy current loss model and analysis has been very mature in the field of motor research [3-5]. There are mainly finite element methods, parameter predictions or experimental tests. However, research in the field of magnetic bearings needs to be improved. Some literature has proposed that the eddy current loss generated by the rotor is proportional to the frequency of the applied current [6-7]. However, the current applied by the RMB is a DC power. In order to reduce the eddy current loss, it is necessary to establish a precise eddy current loss model. David C. Meeker analyzes the rotation loss of a heteropolar radial magnetic bearing [8]. The loss of the rotor during rotation is analyzed by numerical analytical methods. Finally, the correctness of the numerical analysis results is verified by experimental methods. Mizuno et al. tested the magnetic bearing magnetic poles in the NSNS and NNSS configuration with solid rotor iron loss [9]. The test results show that the iron loss in the NSNS magnetic pole distribution is slightly less than that in the NNSS configuration. Sun Jinji analyzed and experimented the eddy current loss of the rotor [10]. In the article, the loss of the rotor was divided into four categories: wind resistance, eddy current loss produced by a radial magnetic bearing, loss caused by axial magnetic bearing, and loss generated by the motor. $\mathrm{Hu}$ Xiaofei [11] analyzed and optimized the eddy current loss of a Homopolar radial magnetic bearing rotor. The influence of the number of magnetic poles on eddy current loss is analyzed. It is concluded that increasing the number of magnetic poles of the magnetic bearing will cause the eddy current loss of the rotor to increase first and then decrease. In addition, the pole width of the magnetic bearing and the increase of the air gap length can effectively reduce the rotor eddy current loss. Based on the analysis results, he proposed a method to reduce the eddy current loss of the magnetic bearing rotor.

This paper presents an analytical model of magnetic pole eddy current loss of a solid rotor of RMB. Considering the difference between the NNNN and NSNS magnetic circuits, the magnetic field expression of 
the stator magnetic pole is discussed. The Fourier series expansion formula is used to substitute the magnetic field of the stator magnetic pole. According to the magnetic field distribution around the magnetic pole surface and the boundary conditions of the rotor surface, the mathematical expression of eddy current loss is obtained. By solving the eddy current loss expression, the eddy current loss value under different arrangement of magnetic poles is obtained. The eddy current loss value of the RMB can be obtained through the free Run-down experiments of the rotor under the vacuum condition. The results of the eddy current loss obtained by the experiment agree well with the numerical solution results. Eddy current loss models and test methods provide theoretical support for analyzing eddy current losses in solid rotors and reducing power consumption.

\section{Analysis of eddy-current loss}

RMB structure shown in Figure 1 (a), mainly including the stator (shown in Figure 1 (b)), stator winding and rotor system. The stator pole adopts the spherical structure, and the rotor pole adopts the spherical structure. This structure can avoid the interference torque caused by the rotor assembly error. The radial magnetic bearing bias flux is generated by the bias current and the control flux is generated by the control current. The rotor is differentially controlled by a bias current and a control current, and is stably suspended in an equilibrium position Magnetic bearing structure parameters are shown in Table 1.

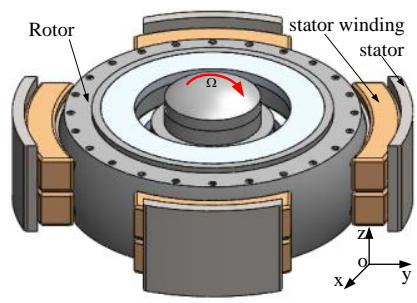

(a)

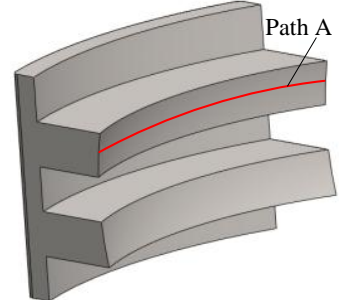

(b)
Figure 1. RMB structure

Table 1. Magnetic bearing structure parameters.

\begin{tabular}{|c|c|}
\hline Design Parameters & Parameter \\
\hline Stator inner diameter $(\mathrm{mm})$ & 158 \\
\hline Stator core circumferential length $(\mathrm{mm})$ & 70 \\
\hline Very wide stator $(\mathrm{mm})$ & 10 \\
\hline Rotor outside diameter $(\mathrm{mm})$ & 157.3 \\
\hline Inner diameter of rotor $(\mathrm{mm})$ & 100 \\
\hline $\begin{array}{c}\text { Distance between stator poles on both sides } \\
(\mathrm{mm})\end{array}$ & 10 \\
\hline Number of turns per coil & 200 \\
\hline $\begin{array}{c}\text { Coil window area (mm*mm)( Radial* } \\
\text { circumferential) }\end{array}$ & $15.5 * 4.5$ \\
\hline Unilateral air gap (mm) & 0.35 \\
\hline Protection air gap (mm) & 0.2 \\
\hline Rotor weight $(\mathrm{kg})$ & 6 \\
\hline Bias current $(\mathrm{A})$ & 0.5 \\
\hline
\end{tabular}

When the rotor rotates at high speed, the magnetic field on the surface of the rotor changes periodically and eddy current loss occurs around the surface of the rotor. This eddy current loss will increase the temperature of the rotor and cause deformation of the rotor structure, which will seriously affect the performance of the rotor. radial magnetic bearing of MSCSG adopts upper and lower double-layer magnetic pole structure to form a closed loop, so the magnetic field distribution of four magnetic poles in each layer generally has two forms, NNNN and NSNS. The eddy current loss produced by these two distributions is different. The eddy current loss analysis of these two structures follows.

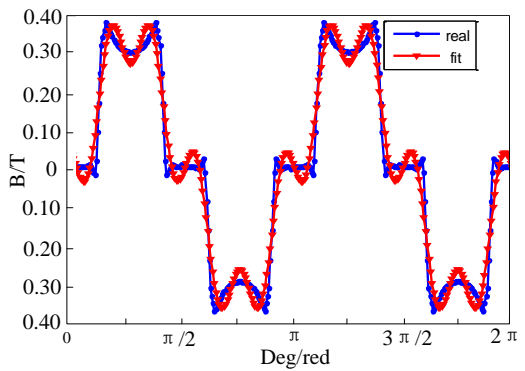

(a) NSNS arrangement

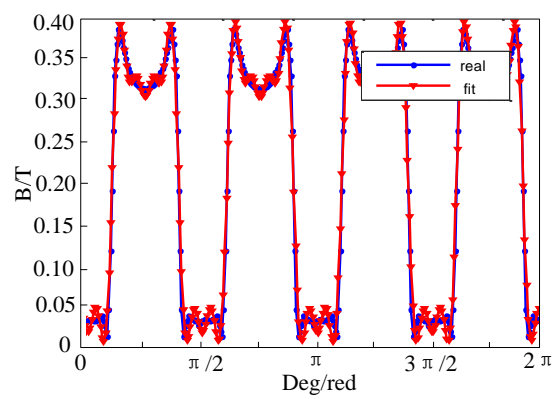

(b) NNNN arrangement

Figure 2. Path A magnetic field distribution

Fig 2 shows the distribution of the magnetic field when the magnetic fields NNNN and NSNS are arranged in the stator magnetic path A (Fig. 1b). The blue lines in the figure represent the real value. Due to the periodic rotation of the rotor, it can be assumed that the rotor surface magnetic field periodically change. From the above figure, we can see that there is a change of magnetic density in the air-gap of the magnetic bearing, and the magnetic flux density of the rotor is unevenly distributed in the circumferential direction. Therefore, when the rotor rotates, the magnetic field on the rotor surface changes and core loss occurs.

Magnetic field of path A expand using Fourier series. The red line represents the result of the Fourier series expansion. The green line According to the fig 2 we can see that the result of Fourier expansion can represent the magnetic field in the path $\mathrm{A}$. The expression of the magnetic field represented by the Fourier series is shown in the following formula (1).

$$
B_{0}=\sum_{n=1}^{\infty} A_{n} \cos (\omega n x)+B_{n} \sin (\omega n x)
$$

According to euler equation, formula(1) can be simplified as the following formula(2). 


$$
B_{0}=\sum_{n=1}^{\infty} C_{n} e^{i \omega T x}+C_{n}^{\prime} e^{-i \omega T x}
$$

For the following calculation, it is assumed that all magnetic poles of RMB are same. In order to solve Maxwell equations, boundary conditions must be defined and boundary conditions are as follows:

1.It is assumed that the magnetic flux density is constant $\left(B_{0}\right)$ at the boundary between the stator and air gap and the applied flux density is periodic.

2. The conductivity and permeability are constant, and the rotor magnetization curve is linear.

3. Magnetic fields and induced currents tend to concentrate on the surface of the rotor. The penetration $\operatorname{depth}(\delta)$ of the magnetic field is much smaller than the diameter of the rotor.

The steady state MAXWELL equation of a moving conductor can be represented by the second-order partial differential equation as follows:

$$
\frac{1}{\mu \delta} \nabla^{2} B+\nabla \times(v \times B)=0
$$

When the rotor rotates at high speed, the polarities of the magnetic poles will change and an alternating magnetic field will be generated in the rotor which will generate eddy current losses.

Compared with the rotor radius, the skin effect depth of the magnetic field is much smaller than the rotor radius. Therefore, the outer surface of the rotor is expanded in the circumferential direction, therefore using the spherical coordinate system instead of Cartesian Cartesian coordinate system produces little error. As shown in Figure 3 is Circumferential expansion diagram of RMB.

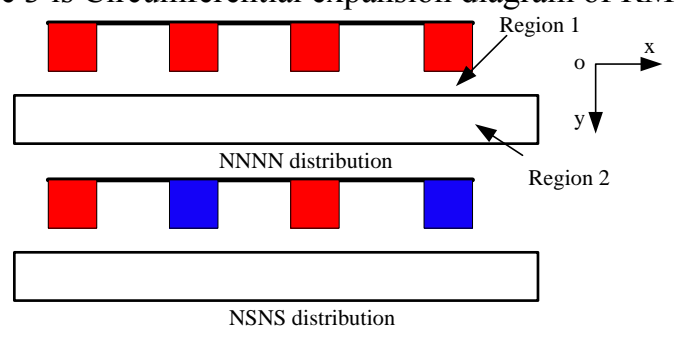

Figure 3. Circumferential expansion diagram

The magnetic field $B$ and the rotor speed $v$ have the following expression in the Cartesian coordinate system:

$$
\begin{gathered}
B=B_{x} \mathbf{i}+B_{y} \mathbf{j}+B_{z} \mathbf{k} \\
v=v_{x} \mathbf{i}
\end{gathered}
$$

In this type of radial magnetic bearing, the magnetic field produces components in the $\mathrm{X}$ and $\mathrm{Y}$ directions and the $\mathrm{Z}$ direction component is 0 . So, $B_{z}=0$, the above equation can be simplified as shown in the following equation:

$$
\left\{\begin{array}{l}
\frac{\partial^{2} B_{x}}{\partial x^{2}}+\frac{\partial^{2} B_{x}}{\partial y^{2}}-\mu \sigma v_{x} \frac{\partial B_{x}}{\partial x}=0 \\
\frac{\partial^{2} B_{y}}{\partial x^{2}}+\frac{\partial^{2} B_{y}}{\partial y^{2}}-\mu \sigma v_{x} \frac{\partial B_{y}}{\partial x}=0
\end{array}\right.
$$

According to the stator magnetic field periodic boundary conditions, the magnetic field produced by the stator magnetic pole can be expressed by the following formula:

$$
B=\left(\begin{array}{c}
B_{x} \\
B_{x}
\end{array}\right)=\left(\begin{array}{c}
\tilde{B}_{x}(y, z) \\
\tilde{B}_{x}(y, z)
\end{array}\right) e^{i k n x}
$$

Because of $\nabla \bullet B=0$, combining (4) (7) lead to

$$
\begin{aligned}
& \frac{\partial^{2} \tilde{B}_{x}}{\partial y^{2}}-q^{2} \tilde{B}_{x}=0 \\
& \frac{\partial^{2} \tilde{B}_{y}}{\partial y^{2}}-q^{2} \tilde{B}_{y}=0
\end{aligned}
$$

In the above formula, $q=\sqrt{k^{2}+k \sigma \mu v_{x}}$, The above differential equation can be obtained:

$$
\begin{aligned}
& \tilde{B}_{x}=-\frac{q}{i k}\left(a e^{k y}-b e^{-k y}\right) \\
& \tilde{B}_{y}=a e^{k y}+b e^{-k y}
\end{aligned}
$$

The magnetic bearing model in the Cartesian coordinate system is divided into two areas. The region 1 is the air gap. At this time, the conductivity $\sigma=0$.so $q=k$. Region 2 is the rotor of the movement. For the result of the above formula 9 in region 1 , the result is:

$$
\begin{aligned}
& B_{x 1}=i e^{i k x}\left(a_{1} e^{k y}-b_{1} e^{-k y}\right) \\
& B_{y 1}=i e^{i k x}\left(a_{1} e^{k y}+b_{1} e^{-k y}\right)
\end{aligned}
$$

The results for region 1 can be simplified as:

$$
\begin{aligned}
& B_{x 2}=b_{2} \frac{q}{i k} e^{i k x} e^{-k y} \\
& B_{y 2}=b_{2} e^{i k x} e^{-k y}
\end{aligned}
$$

According to the boundary conditions, $y=0$, magnetic field is generated by magnetic poles. $y=\Delta$, Here, the magnetic field in Region 1 and Region 2 must be equal. So get the following formula:

$$
\begin{aligned}
& B_{y 1}(\mathrm{y}=0)=c e^{i k x} \\
& B_{y 1}(\mathrm{y}=\Delta)=B_{y 2}(\mathrm{y}=\Delta) \\
& B_{x 1}(\mathrm{y}=\Delta)=\frac{1}{\mu_{r}} B_{x 2}(\mathrm{y}=\Delta)
\end{aligned}
$$

From the formula (12), $a_{1}, b_{1}$ and $b_{2}$ can be obtained. As shown in the formula (13):

$$
\begin{aligned}
& a_{1}=c\left(1-\frac{q}{\mu_{r} k}\right) \frac{e^{-k \Delta}}{2 \gamma} \\
& a_{2}=c\left(1+\frac{q}{\mu_{r} k}\right) \frac{e^{k \Delta}}{2 \gamma} \\
& a_{1}=c \frac{e^{q \Delta}}{\gamma}
\end{aligned}
$$

Where $\gamma=\operatorname{cocosh}(k \Delta)+\frac{q}{\mu_{r} k} \sinh (k \Delta)$

According to the MAXWELL stress tensor, the force acting on the moving conductive rotor is:

$$
\begin{aligned}
& F_{x}=-\frac{1}{\mu_{0}} \int_{s} B_{x} B_{y} d a \\
& F_{x}=-\frac{1}{2 \mu_{0}} \int_{s}\left(B_{x}^{2}-B_{y}^{2}\right) d a
\end{aligned}
$$


The formula (14), $S$ is a closed surface that arbitrarily surrounds a conductor. The face selected in this article is $y=\Delta, 0 \leq x \leq 2 \pi, 0 \leq z \leq b$, Where $z$ is the axial length of the radial magnetic bearing.According to Formula 14, the force applied to the rotor is:

$$
\begin{aligned}
& F_{x}=\frac{i b U}{\mu_{0}} \sum_{n=1}^{\infty} \frac{c_{n} \bar{c}_{n}}{\gamma_{n} \bar{\gamma}_{n}} \frac{q_{n}-\bar{q}_{n}}{\mu_{r} k_{n}} \\
& F_{y}=\frac{b U}{\mu_{0}} \sum_{n=1}^{\infty} \frac{c_{n} \bar{c}_{n}}{\gamma_{n} \bar{\gamma}_{n}} \frac{q_{n} \bar{q}_{n}-\mu_{r}^{2} k_{n}^{2}}{\mu_{r}^{2} k_{n}^{2}}
\end{aligned}
$$

In the above formula $\bar{c}_{n}, \bar{q}_{n}$ and $\bar{\gamma}_{n}$ are the conjugate complex numbers of $c_{n}, q_{n}$ and $\gamma_{n}$. The subscript $\mathrm{n}$ indicates the n-th harmonic of the Fourier transform of the applied magnetic field. The rotor eddy current losses can be obtained by combining Equations 5 and 15. As shown in the following formula:

$$
P=F_{x} v_{x}
$$

\section{Experiment setup and measurement}

The cross-section of the MSCSG prototype is shown in Fig. 4. It mainly consists of RMB ,axial magnetic bearing, LFMB, motor system, rotor and gyro room, as shown in the figure 4.

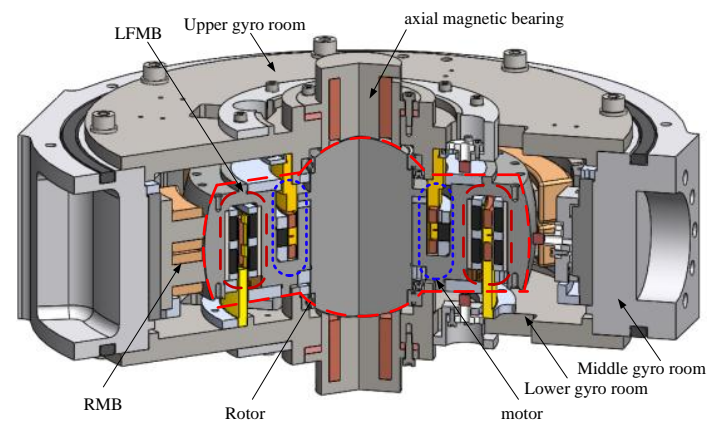

Figure 4. Cross section of the prototyped MSCSG

According to the prototype of the MSCSG, the total rotor losses $\left(P_{t}\right)$ include windage loss $\left(P_{w}\right)$, eddy current loss produced by RMB $\left(P_{r}\right)$ and axial magnetic bearings $\left(P_{a}\right)$, and motor rotation loss $\left(P_{m}\right)$. Therefore, the relationship between various losses can be expressed by the following formula:

$$
P_{t}=P_{w}+P_{r}+P_{a}+P_{m}
$$

The loss of each part of the rotor can be verified by the motor's run-down test. The calculation steps are as follows:

\section{1.windage loss $P_{w}$}

When the MSCSG is evacuated internally, the pressure $\mathrm{P}=0$, and the influence of the wind resistance can be ignored at this time.

\section{2.motor loss $P_{m}$}

The motor used by MSCSG is a brushless DC motor. The magnet is divided into two layers of inner and outer magnets, and the middle of the magnet is a stator of polyimide material. Compared with the traditional structure motor, when the MSCSG rotor rotates at high speed, the magnet and the rotor rotate together, so the magnetic field generated by the motor is invariable in space, so the motor loss is far less than the loss produced by the radial magnetic bearing. So, let's assume the motor loss is equal to $0, P_{m} \approx 0$. Under vacuum conditions, when the rotor is accelerated by the motor to the specified speed, the motor suddenly loses power, and its kinetic energy is mainly converted into magnetic bearing eddy current loss to increase the temperature of the rotor.

\section{Axial magnetic bearing loss $P_{a}$}

Stator magnetic pole of axial magnetic bearing of MSCSG is spherical structure, axial magnetic bearing rotor is spherical structure, when the rotor rotates, the stator of axial magnetic bearing produces a stable magnetic field, So assume the loss caused by the axial magnetic bearing is $P_{a} \approx 0$.

4.Radial magnetic bearing loss

According to the above analysis, the reason for the speed reduction of the rotor only comes from the eddy current loss produced by the RMB. By setting the rotor rundown test, the eddy current loss generated by the RMB can be measured.

When vacuuming the MSCSG, the rotor speed is $\omega$. The kinetic energy of the rotor is:

$$
E_{r}=\frac{1}{2} I \omega^{2}
$$

Where $I$ is the axial moment of inertia of the rotor. According to the above analysis, when the rotor decelerates to 0 , its kinetic energy is mainly converted into heat generated by eddy current losses.so $E_{r}=P_{r} t$. The eddy current loss of the radial magnetic bearing can be calculated by controlling the rotor speed and calculating the time of rotor deceleration. The eddy current loss $\mathrm{p}$ of the RMB is as follows:

$$
P_{r}=\frac{I \omega^{2}}{2 t}
$$

The RMB eddy current loss experiment platform is shown in Figure 2. It Mainly include magnetic MSCSG prototype, vacuum pumping system, magnetic bearing control circuit, and motor control system.

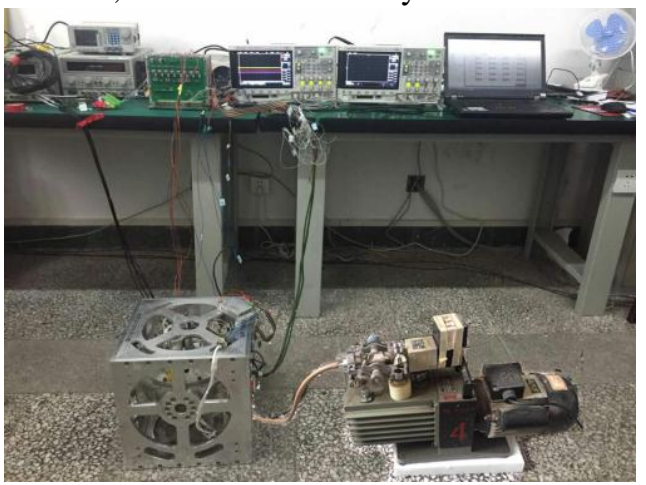

Figure 5. MSCSG experiment platform

The measurement result is shown in Fig 6. According to Figure 6(a), The arrangement of magnetic pole NSNS is 
more obviously than that of NNNN arrangement. Figure 6(b) shows the eddy current loss of the experimental results and the calculated results. The experimental results are basically consistent with the calculation results.

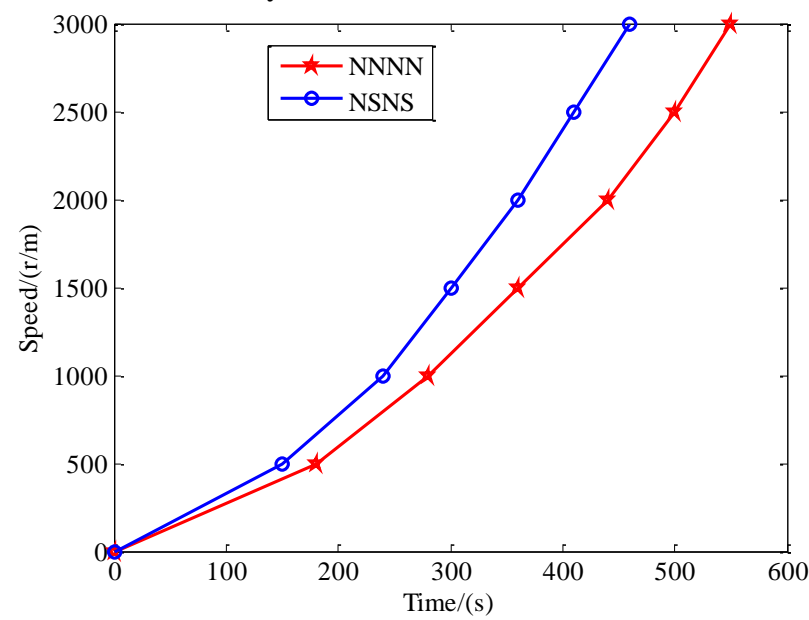

(a) Run-down test performance

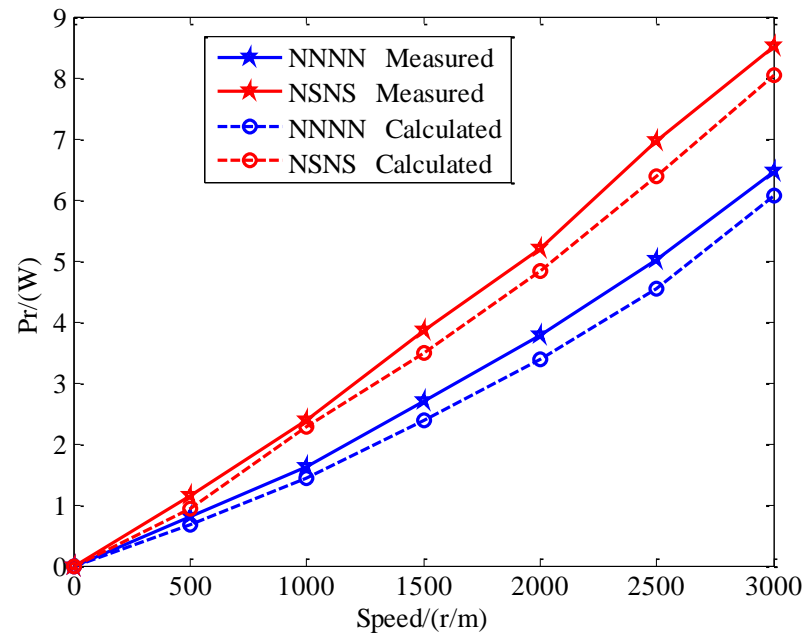

(b) power loss of RMB

Figure 6. Calculated and measured eddy current losses

\section{Conclusion}

This paper presents an eddy current loss analysis model of a solid rotor core magnetic bearing. Considering the difference in the arrangement order of the magnetic poles in the same layer, the magnetic field expression of the rotor core is discussed. According to the magnetic field distribution around the pole surface and the boundary conditions of the rotor core surface, the analytical solutions by solving the electro- magnetic field approach is processed. In the MSCSG prototype, the eddy current loss of the RMB is separated by experimental methods. By comparison, the calculated results is good agreement with the experimental results. The eddy current loss model and test method proposed in this paper provide theoretical support for analyzing the eddy current loss of solid rotors.

\section{References}

1. Le Y, Sun J, Han B. Modeling and Design of 3-DOF Magnetic Bearing for High-Speed Motor Including Eddy-Current Effects and Leakage Effects[J]. IEEE Transactions on Industrial Electronics, 2016, 63(6):3656-3665. Holl, B.G. Orr, Eur. Phys. J. E 14, 7 (2004)

2. J. Fang, S. Zheng, and B. Han, "AMB Vibration Control for Structural Resonance of Double-Gimbal Control Moment Gyro with High-Speed Magnetically Suspended Rotor," IEEE/ASME Trans. Mechatronics, vol.18, no. 1, pp. 32-43, Sep. 2013.Luigi T.De Luca, Propulsion physics (EDP Sciences, Les Ulis, 2009)

3. Dlala E, Belahcen A, Arkkio A. Efficient magnetodynamic lamination model for twodimensional field simulation of rotating electrical machines[J]. Journal of Magnetism \& Magnetic Materials, 2008, 320(20):e1006-e1010.

4. Alatawneh N, Pillay P. Rotational Core Loss and Permeability Measurements in Machine Laminations with Reference to Permeability Asymmetry[J]. IEEE Transactions on Magnetics, 2012, 48(4):1445-1448.

5. Komeza K, Dems M. Finite-Element and Analytical Calculations of No-Load Core Losses in EnergySaving Induction Motors[J]. IEEE Transactions on Industrial Electronics, 2012, 59(7):2934-2946.

6. Bishop J E L. The analysis of eddy-current-limited magnetic domain wall motion, including severe bowing and merging[J]. Journal of Physics D Applied Physics, 1973, 6(6):97.

7. Kendall D, Piercy A R. The frequency dependence of eddy current losses in Terfenol - D[J]. Journal of Applied Physics, 1993, 73(10):6174-6176.

8. Meeker D C, Maslen E H. Prediction of Rotating Losses in Heteropolar Radial Magnetic Bearings[J]. Journal of Tribology, 1998, 120(3):629-635

9. Meeker D C, Filatov A V, Maslen E H. Effect of magnetic hysteresis on rotational losses in heteropolar magnetic bearings[J]. IEEE Transactions on Magnetics, 2004, 40(5):3302-3307.

10. Sun J, Dong C. Analysis and experiment of eddy current loss in Homopolar magnetic bearings with laminated rotor cores[J]. Acta Astronautica, 2013, 89(8):229-235.

11. Hu X F, Liu G, Sun J J, et al. Analysis and optimization of rotor eddy current loss in homopolar radial magnetic bearings[J]. Journal of System Simulation, 2013. 